\title{
First molecular detection of Rickettsia africae in ticks from the Union of the Comoros
}

\author{
Amina Yssouf ${ }^{1}$, Cristina Socolovschi', Tahar Kernif', Sarah Temmam¹, Erwan Lagadec ${ }^{2}$, Pablo Tortosa ${ }^{2,3}$ \\ and Philippe Parola ${ }^{1 *}$
}

\begin{abstract}
Background: Rickettsia africae is the agent of African tick bite fever, a disease transmitted by ticks in sub-Saharan Africa. In Union of the Comoros, a recent study reported the presence of a Rickettsia africae vector but no information has been provided on the circulation of the pathogenic agent in this country.

Methods: To evaluate the possible circulation of Rickettsia spp. in Comorian cattle, genomic DNA was extracted from 512 ticks collected either in the Union of the Comoros or from animals imported from Tanzania and subsequently tested for Rickettsia infection by quantitative PCR.

Results: Rickettsia africae was detected in 90\% (60/67) of Amblyomma variegatum, 1\% (1/92) of Rhipicephalus appendiculatus and 2.7\% (8/296) of Rhipicephalus (Boophilus) microplus ticks collected in the Union of the Comoros, as well as in $77.14 \%$ (27/35) of Amblyomma variegatum ticks collected from imported cattle. Partial sequences of both bacterial $\mathrm{glt} A$ and ompA genes were used in a phylogenetic analysis revealing the presence of several haplotypes, all included within the Rickettsia africae clade.

Conclusions: Our study reports the first evidence of Rickettsia africae in ticks collected from the Union of the Comoros. The data show a significant difference of infection rate of Rickettsia africae infected ticks between the Islands, with maximum rates measured in Grande Comore Island, sheltering the main entry port for live animal importation from Tanzania. The high infection levels reported herein indicate the need for an in-depth assessment of the burden of rickettsioses in the Union of the Comoros, especially among those at risk of infection, such as cattle herders.
\end{abstract}

Keywords: Cattle ticks, Rickettsia africae, Amblyomma variegatum, Rhipicephalus, Comoros

\section{Background}

Tick-borne rickettsioses are considered among the oldest known vector-borne zoonotic diseases; they are caused by obligate intracellular Gram-negative bacteria belonging to the spotted fever group (FSG) of the genus Rickettsia [1]. Many species of this genus are considered to be vertically transmitted symbionts of invertebrates, suggesting that the arthropod vectors act as reservoirs or amplifiers of rickettsiae in the wild [2-4]. In sub-Saharan Africa, several rickettsial strains have been isolated and detected from

\footnotetext{
* Correspondence: philippe.parola@univ-amu.fr

${ }^{1}$ Aix Marseille Université, Unité de Recherche en Maladies Infectieuses et Tropicales Emergentes (URMITE), UM63, CNRS 7278, IRD 198, Inserm 1095, WHO Collaborative Center for Rickettsioses and Other Arthropod-borne Bacterial Diseases, Faculté de Médecine, 27 bd Jean Moulin, 13385 Marseille cedex 5, France

Full list of author information is available at the end of the article
}

ticks and vertebrate animals [5], among which Rickettsia africae, the etiological agent of African tick-bite fever (ATBF), is the most common $[3,6,7]$. The main tick-vectors of Rickettsia africae are Amblyomma hebraeum in southern Africa and Amblyomma variegatum in West, Central and Eastern Africa, as well as in the eastern Caribbean [7-11]. In the Indian Ocean, Rickettsia africae has been previously detected in Amblyomma variegatum in La Reunion and Madagascar [4,12] but never in arthropod and human samples from other islands of the region, including the Comorian Islands.

The Union of the Comoros is composed of three volcanic islands: Grande Comore (the youngest and most elevated island), Anjouan and Moheli. The archipelago is located in the western Indian Ocean, at the northern entrance of the Mozambique Channel between Madagascar 
and the East African coast, and is characterized by a warm and humid tropical climate. Cattle are imported as a food source, mainly from Madagascar. In 2000, a free trade agreement was signed between Comoros and Tanzania, facilitating the reciprocal travel and flow of cattle. Consequently, the export of cattle from Tanzania to Comoros, and particularly to Grande Comore Island, has steadily increased [13]. The likelihood of an increase in the number of pathogens introduced through cattleassociated ticks has also risen.

Recently, an entomological survey carried out on all three islands of the Union of the Comoros showed the presence of Amblyomma variegatum, Rhipicephalus appendiculatus and Rhipicephalus microplus on cattle [13]. Thus, the aim of this study was to detect and determine the prevalence of Rickettsia species that infect ticks on autochthonous cattle and on cattle imported from Tanzania and to evaluate the role of cattle importation in the introduction and of the human pathogen, Rickettsia, throughout the country.

\section{Methods}

\section{Study sites and tick sampling}

The present study used adult ticks that were previously collected to describe cattle tick diversity and distribution in the country. Briefly, the ticks were collected from animals on the three islands of the Union of the Comoros, including 16 of the 17 districts of the country. Adult ticks were collected on cows and goats from the three islands during the 2010 rainy season. For each district, between one and five animals were sampled and identified, and the number of collected ticks was recorded for each site of collection. Following the same protocol, ticks were also collected from cattle imported from Tanzania that were held in the quarantine enclosure located in the harbor vicinity, or in any of the three other quarantine enclosures located in the capital, Moroni (Grande Comore). All ticks were immediately stored in $70 \%$ ethanol until morphological and molecular analyses. Tick species were determined morphologically using standard identification keys [14].

\section{DNA extraction and PCR detection of Rickettsia}

Each tick was sliced longitudinally with a disposable scalpel, and each half was crushed in a buffered solution (G2) with proteinase K (Qiagen Hilden, Germany) and incubated at $56^{\circ} \mathrm{C}$ overnight. Total DNA from half of each tick was extracted in $50 \mu \mathrm{l}$ of eluate using the EZ1 DNA Tissue kit (Qiagen, Hilden, Germany). Rickettsial DNA detection was performed by quantitative PCR using the Eurogentec MasterMix Probe PCR kit (Qiagen, Hilden, Germany) following the manufacturer's instructions with a final volume of $10 \mu \mathrm{l}$ in each reaction as previously described [15]. Each DNA sample was tested by quantitative (q) real-time PCR using a CFX 96 Real
Time System (BIO-RAD, Singapore). The presence of SFG Rickettsia from was determined with a Taqman probe (Eurogentec, Seraing, Germany) and RKND03F and RKND03R specific primers targeting the citrate synthase A $(g l t \mathrm{~A})$ encoding gene [16,17]. Positive samples were subsequently screened with a previously described Rickettsia africae-specific qPCR method. Samples with fewer than 35 cycle thresholds $(\mathrm{Ct})$ were considered positive [18].

In order to generate sequence data allowing phylogenetic analyses of infecting Rickettsia, gltA and ompA were amplified and subsequently sequenced from a subset of randomly selected Rickettsia-positive tick samples. A fragment of gltA gene was amplified using the Rp CS.409d and CS.1258n primers, previously reported to amplify a 750-bp fragment from all known Rickettsia species [6], and ompA gene was targeted by using the primers $190.70,190.180$, and 190.701, amplifying a 629-632-bp fragment of SFG Rickettsia [2,6].

For each PCR reaction, one positive control (Rickettsia montanensis DNA) and 2 negative controls (sterile water containing DNA extracted from uninfected ticks maintained in laboratory colonies) were included, with the exception of the Rickettsia africae-specific qPCR reaction in which Rickettsia africae DNA was used as positive control.

The resulting PCR products were purified and directly sequenced using a BigDye Terminator Cycle Sequencing Kit (Perkin Elmer Applied Biosystems, USA) and an ABI PRISM automated sequencer (Applied Biosystems, USA), as previously described [6].

\section{Sequence editing and phylogenetic analyses}

Sequences were analyzed using Chromas Pro (version 1.49 beta Technelysium Pty Ltd, Tewantin, Australia) and compared with sequences available in the GenBank database using NCBI BLAST. Multiple sequence alignments were noted at the nucleotide and amino acid levels using ClustalW implemented in BioEdit software. The sequences matched with sequences from other glt $A$ and $о m p A$ rickettsiae retrieved from the GenBank database. Rickettsiaconorii and Rickettsia rickettsii sequences have been chosen as root sequences. Accession numbers of the GenBank sequences used for the genetic analyses are indicated in the phylogenetic trees. The selection of the DNA substitution model that best fit the data was performed with MEGA 5.2 and was considered for phylogenetic analyses. We selected different models of nucleotide substitution using the corrected Akaike information criterion. Bayesian phylogenetic inference (BI) was carried out using Mr Bayes 3.1.2 [19] with two independent runs of four incrementally heated, Metropolis Coupled Markov chain Monte Carlo (MC) inquiries beginning with a random tree. $\mathrm{MC}$ queries were run for 
$1,000,000$ generations with trees and associated model parameters sampled every 200 generations. The initial 1000 trees in each run were discarded as burn-in samples, and the harmonic mean of the likelihood was calculated by combining each of the two independent runs.

\section{Statistical analysis}

A statistical analysis was performed to compare the prevalence of Rickettsia africae between the islands using the Mantel-Haenszel test implemented in Epi-info version 3.5 followed by the Yates correction option. Differences were considered statistically significant for $\mathrm{P}$ values $<0.05$.

\section{Results}

\section{PCR detection of rickettsia}

\section{Ticks collected on autochthonous cattle}

Rickettsial DNA was detected in 14\% (67/477) of ticks from the Union of the Comoros. Table 1 presents the distribution of samples positive for Rickettsia by tick species and location. The mean $\mathrm{Ct} \pm \mathrm{SD}$ value of glt $\mathrm{A}$ amplification by qPCR of positive tick samples was $28.34 \pm 3.11$. All positive samples were also positive for an Rickettsia africae-specific qPCR with a mean $\mathrm{Ct} \pm \mathrm{SD}$ value of $28.51 \pm 2.03$. On Grande Comore Island, 90\% (60/67) of Amblyomma variegatum ticks tested positive for Rickettsia africae, with Ct values averaging $28.67 \pm$ 1.95, while $1.9 \%$ (2/105) of Rhipicephalus spp. ticks were positive, with a Ct averaging $28.88 \pm 1.8$ (Figure 1). The prevalence of Rickettsia africae on Anjouan Island was $8.12 \%(13 / 160)$, and a significant difference in prevalence was observed between Anjouan and Grande Comore $32.48 \%(51 / 157)$ (Mantel-Haenszel test, P value $<0.001$ ). Among the positive samples from Anjouan, 61.5\% (8/13) were obtained from Amblyomma variegatum ticks, while the remainders were from Rhipicephalus microplus (Table 1). In Mohéli, one of the five regions we visited had cattle carrying infected ticks (Figure 1). The prevalence of Rickettsia africae on this island was 1.9\% (3/160), which was also significantly different from that found on Grande Comore (Mantel-Haenszel test, P-value <0.001) and Anjouan (Mantel-Haenszel test, P-value $=0.016$ ). All positive ticks from Mohéli were Amblyomma variegatum (Table 1), with positive PCRs displaying an average Ct of $25.24 \pm 1.97$.
The amplification, sequencing and BLAST analyses of ompA and gltA genes from positive DNA samples extracted from Amblyomma variegatum, Rhipicephalus microplus and Rhipicephalus appendiculatus ticks confirmed the presence of Rickettsia africae in 14\% of our samples. The sequence analysis of ompA genes obtained from Rhipicephalus spp. samples revealed 99.0 to $99.6 \%$ nucleotide identity with Rickettsia africae detected from Rhipicephalus evertsi evertsi in Senegal (GenBank accession numbers JN043509), while $R$. africae sequenced from Amblyomma variegatum showed 98 to $99.7 \%$ average identity with $R$. africae detected in Ethiopia from the same tick species (GenBank accession numbers CP001612). The sequencing of gltA genes obtained from Amblyomma variegatum and Rhipicephalus spp. showed 99.1 to $99.42 \%$ average identity with the published sequences of Rhipicephalus africae that were amplified from Amblyomma variegatum collected in the West Indies (GenBank accession number HM050288).

\section{Ticks collected from imported cattle}

Of the ticks collected from cattle imported from Tanzania, $77.14 \%$ (27/35) were positive for Rickettsia spp., with a Ct average of $27.75 \pm 4.13$. All of the samples detected positive for Rickettsia spp. were also positive for Rickettsia africae by qPCR (Figure 1), with Ct averages of $26.7 \pm 4.67$.

The sequencing of ompA genes obtained from Amblyomma variegatum collected from imported cattle showed an average of $98 \%$ alignment with published sequences of Rickettsia africae from Amblyomma variegatum collected in Antigua (GenBank accession number EU622980).

The sequence analysis of the $g l t A$ gene obtained from Amblyomma variegatum ticks revealed 98.0 to $99.0 \%$ nucleotide identity with the gltA gene from Rickettsia africae detected in Amblyomma variegatum from Ethiopia (GenBank accession numbers CP001612) and in Rhipicephalus evertsi evertsi from Senegal (GenBank accession numbers HM050288), respectively.

\section{Phylogenetic analysis}

The best DNA substitution model fitting the data was determined to be HKY for both the gltA and ompA sequences. The Bayesian Inference tree based on gltA sequences

Table 1 Prevalence of Rickettsia africae by species and regions

\begin{tabular}{lcccc}
\hline Species & $\begin{array}{c}\text { Amblyomma } \\
\text { variegatum }\end{array}$ & $\begin{array}{c}\text { Rhipicephalus } \\
\text { microplus }\end{array}$ & $\begin{array}{c}\text { Rhipicephalus } \\
\text { appendiculatus }\end{array}$ & $\begin{array}{c}\text { Number of ticks } \\
\text { tested by region }\end{array}$ \\
\hline Grande Comore & $94.23 \%(49 / 52)$ & $8 \%(1 / 13)$ & $1 \%(1 / 92)$ & 157 \\
Anjouan & $89 \%(8 / 9)$ & $3.3 \%(5 / 151)$ & - & 160 \\
Moheli & $7 \%(3 / 28)$ & $0 \%(0 / 132)$ & - & 160 \\
Ticks collected from imported cattle & $77.14 \%(27 / 35)$ & - & $1 \%(1 / 95)$ & 35 \\
Total from the Union of the Comoros & $65.17 \%(58 / 89)$ & $2.7 \%(8 / 296)$ & 477 \\
\hline
\end{tabular}




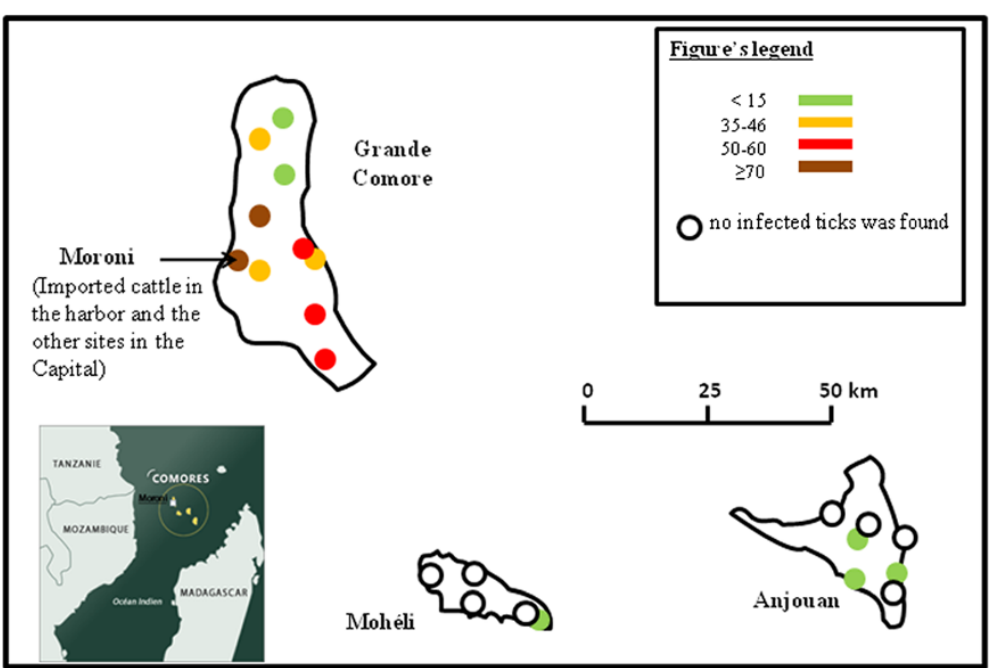

Figure 1 Prevalence of infected ticks by island and regions.

(Figure 2A) showed a distinct phylogenetic Comorian clade (posterior probability $>0.968$ ) in which one well-supported group of sequences (posterior probability $>0.999$ ) included sequences from Rickettsia africae infecting ticks sampled in Grande Comore and Anjouan but not Mohéli, and a Rickettsia africae sequence obtained from a tick imported from Tanzania. The Bayesian Inference tree based on ompA sequences (Figure 2B) showed a similar tree topology, which consolidate our analysis.
Consensus sequences of Rickettsia africae infecting ticks sampled on Grande Comore, Mohéli, Anjouan and Tanzania were generated and aligned to determine nucleotide mutations specific to a geographic origin. No specific mutation was observed for the ompA genes (data not shown); interestingly, the gltA sequences originating from Mohéli have 5 specific non-synonymous mutations (Table 2). The same analysis was conducted to construct consensus sequences of Rickettsia africae isolated from

\section{A}

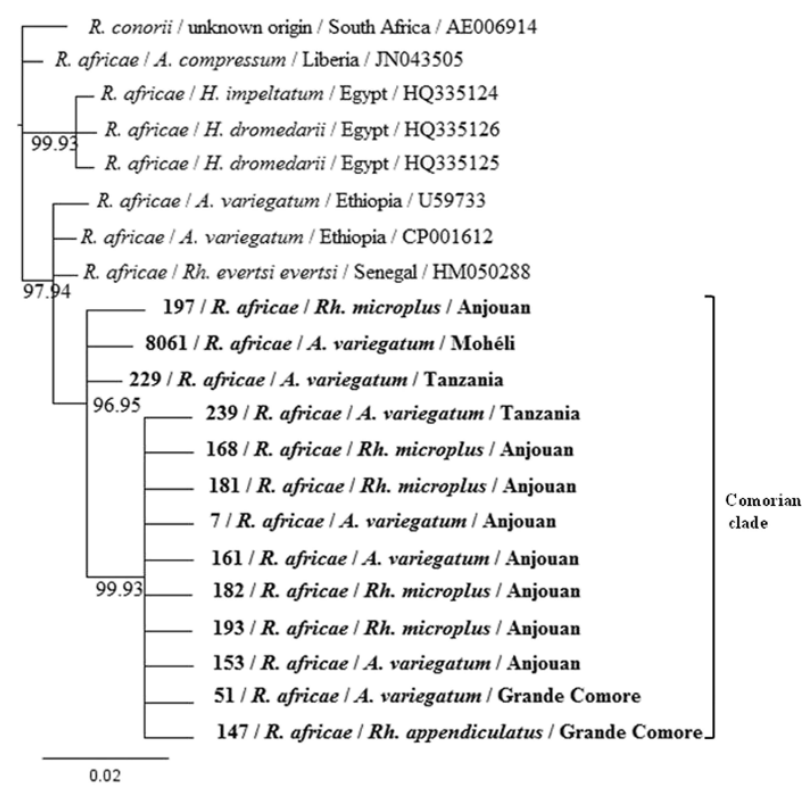

B

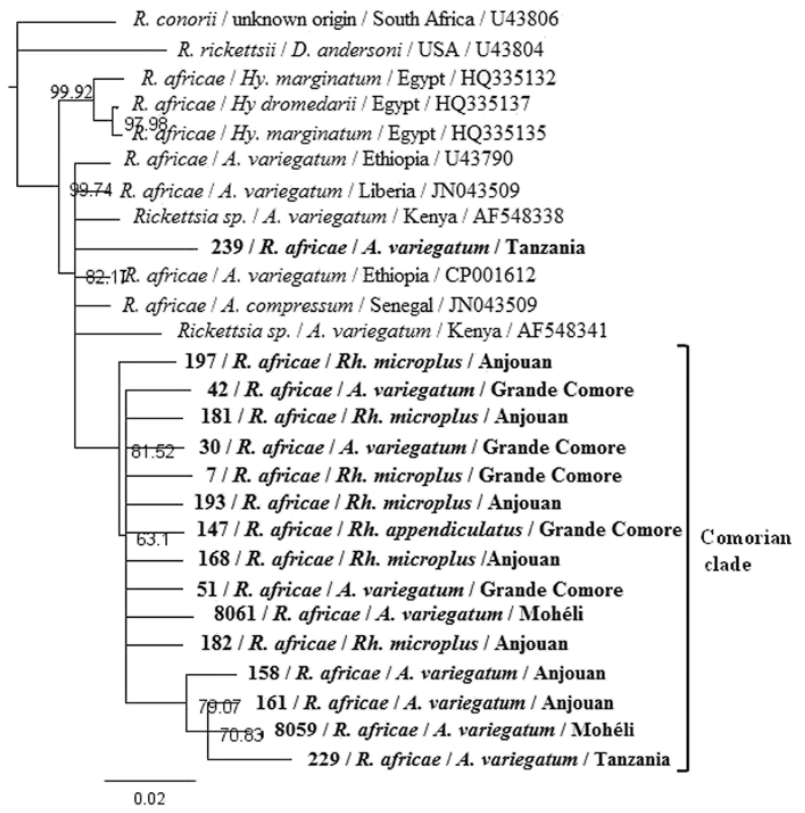

Figure 2 Phylogenetic tree inferred from the comparison of Rickettsia africae strains from specimens tested and selected GenBank Rickettsia africae sequences. Posterior probabilities are expressed in percentages and indicated at branch nodes. The sequences generated in this study are highlighted in bold. A: Phylogenetic tree based on the gltA citrate synthase-encoding gene. B: Phylogenetic tree based on the ompA outer membrane protein A gene. Abbreviation: $R h=$ Rhipicephalus; Hy = Hyalomma; $D=$ Dermacentor. 
Table 2 Non-synonymous mutations in Rickettsia africae gltA Mohéli sequences

\begin{tabular}{|c|c|c|c|c|c|}
\hline Nucleotide position (U59733) & 560 & 566 & 635 & 692 & 1067 \\
\hline Codon sequence & GAT $\rightarrow$ AAT & $\mathrm{CCG} \rightarrow \mathrm{TCG}$ & $\mathrm{CCA} \rightarrow \mathrm{GCA}$ & $\mathrm{AAT} \rightarrow \mathrm{TAT}$ & $\mathrm{AGA} \rightarrow \mathrm{GGA}$ \\
\hline Amino-acid mutation in Mohéli gltA sequence & $\mathrm{D} \rightarrow \mathrm{N}$ & $P \rightarrow S$ & $\mathrm{P} \rightarrow \mathrm{A}$ & $N \rightarrow Y$ & $R \rightarrow G$ \\
\hline
\end{tabular}

distinct tick species, but no specific mutation was observed in either the ompA or gltA gene (data not shown).

\section{Discussion}

A. variegatum is the main vector of Rickettsia africae, a spotted fever group (SFG) Rickettsia bacterium in subSaharan Africa [9,20,21], though it is also considered a competent vector for other human and animal pathogens [22], including the highly virulent Crimean-Congo hemorrhagic fever virus [23]. In the present study, we show for the first time the presence of Rickettsia africae in cattle ticks collected from the Union of the Comoros. The confirmation of the presence of this Rickettsia spp. provides background for further epidemiologic and clinical investigations of tick-borne diseases in the Union of the Comoros. Indeed, other than Theileria parva, a parasitic protozoan that is the causative agent of the East Coast fever in cattle and that was previously detected in Rhipicephalus appendiculatus [24], no other tick-borne pathogens have been detected in this country.

We provide evidence for Rickettsia africae infection in Amblyomma variegatum ticks from all three islands of the Union of the Comoros. Although the Amblyomma variegatum tick infection rate varied among the islands as observed in Grande Comore and Anjouan, further study needs to confirm this result. The presence of Rickettsia africae-infected ticks in Grande Comore and Anjouan is congruent with the geographic distribution of Amblyomma variegatum [8]. The high prevalence of Rickettsia africae (90\%) in Amblyomma variegatum collected in all study sites of Grande Comore showed endemicity of this bacterium and that this tick species is the reservoir of $R$. africae in the Archipelago. Ticks of the genus Amblyomma are considered to be the main vectors for Rickettsia africae although this bacterium has recently been found infecting other genera, including Rhipicephalus [21,22,25]. In this study, we also found Rhipicephalus spp. ticks carrying Rickettsia africae DNA although the presence rate was substantially lower (2\%) than the rate measured in Amblyomma variegatum (65.17\%). However, our data do not provide direct evidence for the vector competence of the Rhipicephalus (Boophilus) microplus genus for Rickettsia africae because Rhipicephalus-positive ticks were always collected on animals infested with $A$. variegatum ticks that tested positive for $R$. africae. Thus, we can hypothesize that Rhipicephalus ticks that were positive for Rickettsia africae acquired the pathogen during a blood meal, but this does not prove that these species of ticks are competent vectors for Rickettsia africae. Further experimental evaluations of vector competence clearly need to be carried out in order to establish the vector competence of Rhipicephalus ticks.

Ticks sampled on cattle imported from Tanzania showed a prevalence of $77.14 \%$ for Rickettsia africae, which is coherent with serological studies previously carried out in Tanzania that also showed high seroprevalence levels [26]. Rickettsia africae polymorphism did not show any island or tick host species structuration. Comorian haplotypes were closely related with African haplotypes, thus strengthening the hypothesis of an African origin for Rickettsia africae in the Comoros archipelago. Phylogenetic trees could not determine the origin of Rickettsia africae infection in the ticks imported from Tanzanian cattle. However, the polymorphism of ompA and gltA is notoriously weak and sequencing of additional markers may help in resolving this issue. Citrate synthase is a component of nearly all living cells and is one enzyme of the citric acid cycle, a key metabolic pathway that plays a key role in energy production [27]. The $O m p A$ gene plays a role in a protective immune response and is considered as a good candidate for phylogenetic analysis for most of the SFG Rickettsiae [2]. These gene sequences showed no clear differences between east African and Comorian bacterial haplotypes, thus supporting an African/Comorian Rickettsia africae metapopulation, although the use of additional markers, or the full sequencing of bacterial isolates would obviously provide more robust information.

\section{Conclusion}

The PCR assays and sequence analyses provide new information on the epidemiology of ticks infected with Rickettsia africae in the Union of the Comoros. The detection of Rickettsia africae in ticks collected from live cattle imported from Tanzania represents a risk to local farms. Our results strengthen the need for an evaluation of the burden of ATBF in the human populations in contact with cattle. Together with the previous investigation reporting the introduction of a tick species of veterinary importance new to the country, the present study clearly shows that because of the insularity, ticks and parasites introduction into the country represents a risk of biological invasion. Therefore, increased vigilance is required to limit this risk [28].

\section{Competing interests}

The authors declare that they have no competing interests. 


\section{Authors' contributions}

AY performed the experiments and drafted the manuscript with the input from all co-authors, CS provided constant intellectual support in the course of the experiments, TK participated in the experiments. ST performed the phylogenetic analysis, EL provided the biological material. PT conceived the study. PP conceived the study and gave approval of the final version. All authors read and approved the final version of the manuscript.

\section{Acknowledgements}

We wish to thank Frederic Stachurski for his contribution in the achievement of the prevalence distribution map of Rickettsia africae in the Union of the Comoros.

\section{Author details}

'Aix Marseille Université, Unité de Recherche en Maladies Infectieuses et Tropicales Emergentes (URMITE), UM63, CNRS 7278, IRD 198, Inserm 1095, WHO Collaborative Center for Rickettsioses and Other Arthropod-borne Bacterial Diseases, Faculté de Médecine, 27 bd Jean Moulin, 13385 Marseille cedex 5 , France. ${ }^{2}$ Centre de Recherche et de Veille sur les Maladies Emergentes dans I'Océan Indien (CRVOI)-Plateforme de Recherche CYROI- 2, rue Maxime Rivière, 97490 Ste Clotilde, La Reunion, France. ${ }^{3}$ Université de La Réunion, Ste Clotilde, La Réunion, France.

Received: 4 March 2014 Accepted: 9 September 2014

Published: 22 September 2014

\section{References}

1. Parola P, Paddock CD, Socolovschi C, Labruna MB, Mediannikov O, Kernif T, Abdad MY, Stenos J, Bitam I, Fournier PE, Raoult D: Update on tick-borne rickettsioses around the world: a geographic approach. Clin Microbiol Rev 2013, 26:657-702.

2. Fournier PE, Roux V, Raoult D: Phylogenetic analysis of spotted fever group rickettsiae by study of the outer surface protein rOmpA. Int I Syst Bacteriol 1998, 48(Pt 3):839-849.

3. Parola P, Vestris G, Martinez D, Brochier B, Roux V, Raoult D: Tick-borne rickettiosis in Guadeloupe, the French West Indies: isolation of Rickettsia africae from Amblyomma variegatum ticks and serosurvey in humans, cattle, and goats. Am J Trop Med Hyg 1999, 60:888-893.

4. Parola P, Barre N: Rickettsia africae, the agent of African tick-bite fever: an emerging pathogen in the West Indies and reunion island (Indian ocean). Bull Soc Pathol Exot 2004, 97:193-198.

5. Parola P: Rickettsioses in sub-Saharan Africa. Ann N Y Acad Sci 2006, 1078:42-47.

6. Mura A, Socolovschi C, Ginesta J, Lafrance B, Magnan S, Rolain JM, Davoust B, Raoult D, Parola P: Molecular detection of spotted fever group rickettsiae in ticks from Ethiopia and Chad. Trans $R$ Soc Trop Med Hyg 2008, 102:945-949.

7. Ndip LM, Fokam EB, Bouyer DH, Ndip RN, Titanji VP, Walker DH, McBride JW: Detection of Rickettsia africae in patients and ticks along the coastal region of Cameroon. Am J Trop Med Hyg 2004, 71:363-366.

8. Eldin C, Mediannikov O, Davoust B, Cabre O, Barre N, Raoult D, Parola P: Emergence of Rickettsia africae, Oceania. Emerg Infect Dis 2011, 17:100-102.

9. Kelly PJ: Rickettsia africae in the West Indies. Emerg Infect Dis 2006, 12:224-226.

10. Jensenius M, Fournier PE, Kelly P, Myrvang B, Raoult D: African tick bite fever. Lancet Infect Dis 2003, 3:557-564.

11. Barre N, Garris G, Camus E: Propagation of the tick Amblyomma variegatum in the Caribbean. Rev Sci Tech 1995, 14:841-855.

12. Fournier PE, El Karkouri K, Leroy Q, Robert C, Giumelli B, Renesto P, Socolovschi C, Parola P, Audic S, Raoult D: Analysis of the Rickettsia africae genome reveals that virulence acquisition in rickettsia species may be explained by genome reduction. BMC Genomics 2009, 10:166.

13. Yssouf A, Lagadec E, Bakari A, Foray C, Stachurski F, Cardinale E, Plantard O, Tortosa P: Colonization of Grande Comore Island by a lineage of Rhipicephalus appendiculatus ticks. Parasit Vectors 2011, 4:38.

14. Walker AR, Bouattour A, Camicas J, Estrada-Pena A, Horak IG, Pegram RG, Preston PM: Ticks of Domestic Animals in Africa: A Guide to Identification of Species, Bioscience Reports. Edinburgh, United Kingdom: 2003.

15. Socolovschi C, Pages F, Raoult D: Rickettsia felis in Aedes albopictus mosquitoes, Libreville, Gabon. Emerg Infect Dis 2012, 18:1687-1689.
16. Bechah $Y$, Socolovschi C, Raoult D: Identification of rickettsial infections by using cutaneous swab specimens and PCR. Emerg Infect Dis 2011, 17:83-86.

17. Socolovschi C, Gomez J, Marie JL, Davoust B, Guigal PM, Raoult D, Parola P: Ehrlichia canis in Rhipicephalus sanguineus ticks in the ivory coast. Ticks Tick Borne Dis 2012, 3:411-413.

18. Kernif T, Djerbouh A, Mediannikov O, Ayach B, Rolain JM, Raoult D, Parola P, Bitam I: Rickettsia africae in Hyalomma dromedarii ticks from sub-Saharan Algeria. Ticks Tick Borne Dis 2012, 3:377-379.

19. Ronquist F, Huelsenbeck JP: MrBayes 3: Bayesian phylogenetic inference under mixed models. Bioinformatics 2003, 19:1572-1574.

20. Lynen G, Zeman P, Bakuname C, Di Giulio G, Mtui P, Sanka P, Jongejan F: Cattle ticks of the genera Rhipicephalus and amblyomma of economic importance in Tanzania: distribution assessed with GIS based on an extensive field survey. Exp Appl Acarol 2007, 43:303-319.

21. Macaluso KR, Davis J, Alam U, Korman A, Rutherford JS, Rosenberg R, Azad AF: Spotted fever group rickettsiae in ticks from the Masai Mara region of Kenya. Am J Trop Med Hyg 2003, 68:551-553.

22. Mediannikov O, Diatta G, Zolia Y, Balde MC, Kohar H, Trape JF, Raoult D: Tick-borne rickettsiae in Guinea and Liberia. Ticks Tick Borne Dis 2012, 3:43-48.

23. Camicas JL, Cornet JP, Gonzalez JP, Wilson ML, Adam F, Zeller HG: CrimeanCongo hemorrhagic fever in Senegal. Latest data on the ecology of the CCHF virus. Bull Soc Pathol Exot 1994, 87:11-16.

24. De Deken R, Martin V, Saido A, Madder M, Brandt J, Geysen D: An outbreak of east coast fever on the Comoros: a consequence of the import of immunised cattle from Tanzania? Vet Parasitol 2007, 143:245-253.

25. Portillo A, Perez-Martinez L, Santibanez S, Blanco JR, Ibarra V, Oteo JA: Detection of Rickettsia africae in Rhipicephalus (Boophilus) decoloratus ticks from the Republic of Botswana, south Africa. Am J Trop Med Hyg 2007, 77:376-377.

26. Prabhu M, Nicholson WL, Roche AJ, Kersh GJ, Fitzpatrick KA, Oliver LD, Massung RF, Morrissey AB, Bartlett JA, Onyango JJ, Maro VP, Kinabo GD, Saganda W, Crump JA: Q fever, spotted fever group, and typhus group rickettsioses among hospitalized febrile patients in northern Tanzania. Clin Infect Dis 2011, 53:e8-e15.

27. Roux V, Rydkina E, Eremeeva M, Raoult D: Citrate synthase gene comparison, a new tool for phylogenetic analysis, and its application for the rickettsiae. Int I Syst Bacteriol 1997, 47:252-261.

28. Tortosa P, Pascalis H, Guernier V, Cardinale E, Le Corre M, Goodman SM, Dellagi K: Deciphering arboviral emergence within insular ecosystems. Infect Genet Evol 2012, 12:1333-1339.

doi:10.1186/1756-3305-7-444

Cite this article as: Yssouf et al:: First molecular detection of Rickettsia africae in ticks from the Union of the Comoros. Parasites \& Vectors 2014 7:444

\section{Submit your next manuscript to BioMed Central and take full advantage of:}

- Convenient online submission

- Thorough peer review

- No space constraints or color figure charges

- Immediate publication on acceptance

- Inclusion in PubMed, CAS, Scopus and Google Scholar

- Research which is freely available for redistribution 